\title{
On Lie Algebras of Vector Fields on Smooth Orbifolds
}

By

\author{
KŌJUN ABE*
}

\section{§0. Introduction}

In the previous paper [1], we studied Pursell-Shanks type theorem for orbit spaces of $G$-manifolds. The purpose of this paper is to prove that this still holds for smooth orbifolds and for fibrations over smooth orbifolds with compact generic fibre.

Let $B$ and $B^{\prime}$ be connected smooth orbifolds. Let $\mathscr{D}(B)$ (resp. $\mathfrak{X}(B)$ ) be the Lie algebra of all smooth vector fields (resp. strata preserving smooth vector fields) on $B$ with compact support.

Theorem 0.1. The following statements are equivalent.

(1) There exists a Lie algebra isomorphism $\Phi: \mathfrak{D}(B) \rightarrow \mathfrak{D}\left(B^{\prime}\right)$.

(2) There exists a Lie algebra isomorphism $\Phi: \mathfrak{X}(B) \rightarrow \mathfrak{X}\left(B^{\prime}\right)$.

(3) There exists a diffeomorphism $\sigma: B \rightarrow B^{\prime}$.

$E$ admits a natural orbifold structure. Let $p: E \rightarrow B$ (resp. $\left.p^{\prime}: E^{\prime} \rightarrow B^{\prime}\right)$ be a fibration over $B$ (resp. $B^{\prime}$ ) with generic fibre $F$ (resp. $F^{\prime}$ ), a connected closed smooth manifold. Let $\mathscr{D}(E ; p)($ resp. $\mathfrak{X}(E: p)$ ) be the subalgebra of $\mathfrak{D}(E)$ (resp. $\mathfrak{X}(E)$ ) consisting of fibration preserving vector fields (see $\S 1$ ). Using Theorem 0 . 1, we prove the following.

Theorem 0.2. The following statements are equivalent.

(1) There exists a Lie algebra isomorphism $\Phi: \mathscr{D}(E ; p) \rightarrow \mathscr{D}\left(E^{\prime} ; p^{\prime}\right)$.

(2) There exists a Lie algebra isomorphism $\Phi: \mathfrak{X}(E ; p) \rightarrow \mathfrak{X}\left(E^{\prime} ; p^{\prime}\right)$.

(3) There exists a fibration preserving diffeomorphism $\sigma: E \rightarrow E^{\prime}$ 。

Communicated by N. Shimada, September 10, 1985.

* Department of Mathematics, Faculty of Liberal Arts, Shinshu University, Matsumoto, 390, Japan. 
Theorem 0.1 is a special case of Theorem 0.2 , but we prove Theorem 0.2 by using Theorem 0.1 . Theorem 0.2 was proved by Omori [6] for the case that $E$ and $E^{\prime}$ are smooth fibre bundles.

The paper is organized as follows. In $\S 1$ we define smooth vector fields on smooth orbifolds and on fibrations over smooth orbifolds. $\S 2$ is devoted to preliminaries. In $\S 3$ and $\S 4$ we determine the maximal ideals of $\mathfrak{X}(B), \mathfrak{X}(E ; p)$ and some subalgebras of $\mathfrak{X}(E ; p)$. In $\S 5$ we prove that Theorem 0.2 (2) implies (3). In $\S 6$ and $\S 7$ we determine the maximal ideals of $\mathscr{D}(B)$ and $\mathscr{D}(E ; p)$, and prove Theorem 0.1 and Theorem 0.2.

The author would like to thank the referee for reading the paper in detail and giving him valuable suggestions.

\section{§1. Definitions}

In this section we shall define smooth vector fields on smooth orbifolds and fibrations over smooth orbifolds.

Definition 1.1 (See Satake [7] and Thurston [10])

A paracompact Hausdorff space $M$ is called a smooth orbifold if there exists an open covering $\left\{U_{i}\right\}$ of $M$, closed under finite intersections, satisfying the following conditions.

(1) For each $U_{i}$, there are a finite group $\Gamma_{i}$, a smooth action of $\Gamma_{i}$ on an open set $\tilde{U}_{i}$ of $\mathrm{R}^{n}$ and a homeomorphism $\phi_{i}: U_{i} \rightarrow$ $\tilde{U}_{i} / \Gamma_{i}$.

(2) Whenever $U_{i} \subset U_{j}$, there is a smooth embedding $\phi_{i j}: \tilde{U}_{i} \rightarrow \tilde{U}_{j}$ such that the following diagram commutes:

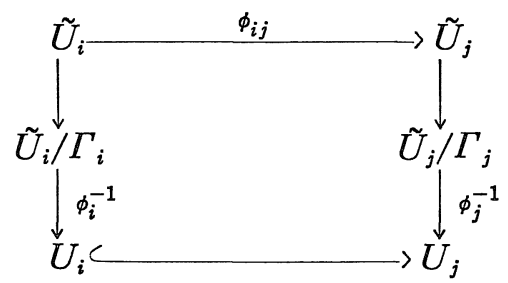

Remark. We can choose the finite group $\Gamma_{i}$ such that the action $\Gamma_{i} \times \tilde{U}_{i} \rightarrow \tilde{U}_{i}$ is effective, and then there is a unique injective group homomorphism $f_{i j}: \Gamma_{i} \rightarrow \Gamma_{j}$ such that $\phi_{i j}$ is equivariant with respect 
to $f_{i j}$.

Two coverings give rise to the same orbifold structure if they can be combined consistently to give a larger cover still satisfying the conditions.

Let $F$ be a smooth manifold. A paracompact Hausdorff space $E$ with a continuous map $p: E \rightarrow M$ is called a smooth fibrations over a smooth orbifold $M$ with generic fibre $F$ if the following conditions are satisfied.

(1) For each $U_{i}$, there exists a smooth $\Gamma_{i}$-action on $F$ such that $p^{-1}\left(U_{i}\right)=U_{i} \times F / \Gamma_{i}$ and the following diagram commutes:

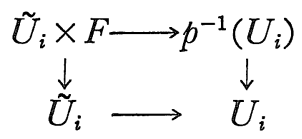

(2) Whenever $U_{i} \subset U_{j}$, the reexists a smooth embedding $\phi_{i j}: \tilde{U}_{i} \times$ $F \rightarrow \tilde{U}_{j} \times F$ such that the following diagram commutes:

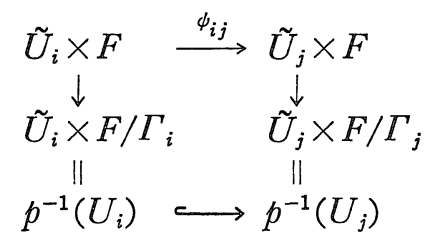

Definition $\mathbb{1}_{0} 2$ (See $[1], \S 1$ ) Let $M$ be a smooth orbifold given in Definition 1.1, A function $f: M \rightarrow R$ is said to be smooth if the composition $\tilde{U}_{i} \rightarrow \tilde{U}_{i} / \Gamma_{i}=U_{i} \stackrel{f}{\rightarrow} R$ is smooth for any $U_{i \circ}$ Let $C^{\infty}(M)$ denote the algebra of all smooth functions on $M$. As in [1], §1 for any $b \in M$ we can define a tangent space $\tau_{b}(M)$. We define a smooth vector field on $M$ to be a real linear derivation on $C^{\infty}(M)$. Let $D(M)$ be the Lie algebra of all smooth vector fields on $M$ with compact support. A smooth vector field $X$ on $M$ is called strata preserving if $X$ preserves the ideals in $C^{\infty}(M)$ of smooth functions which vanish on the strata of $U_{i}=\tilde{U}_{i} / \Gamma_{i}$ for any $U_{i}$ (cf. [10], $\S 2$ )。 Let $\mathfrak{X}(M)$ denote the Lie algebra of strata preserving vector fields on $M$ with compact support.

Let $p: E \rightarrow M$ be a smooth fibration over a smooth orbifold $M$ with generic fibre $F$. Then $E$ has a natural smooth orbifold structure. Let $X \in \mathfrak{X}(E) . \quad X$ is said to be fibration preserving if $(d p)_{e_{1}}\left(X_{e_{1}}\right)=$ $(d p)_{e_{2}}\left(X_{e_{2}}\right)$ for any $b \in M$ and $e_{1}, \mathrm{e}_{2} \in p^{-1}(b)$. Let $\mathfrak{X}(E ; p)=\{X \in \mathfrak{X}(E) ; X$ 
is fibration preserving\}. Let $p^{\prime}: E^{\prime} \rightarrow M^{\prime}$ be another smooth fibration over a smooth orbifold $M^{\prime}$ with generic fibre $F^{\prime}$. We say a map $\sigma: E \rightarrow E^{\prime}$ is smooth if $f \circ \sigma \in C^{\infty}(E)$ for any $\mathrm{f} \in C^{\infty}\left(E^{\prime}\right)$, and $\sigma$ is diffeomorphic if $\sigma$ and $\sigma^{-1}$ are smooth. For a diffeomorphism $\sigma: E \rightarrow E^{\prime}$, let $\sigma_{*}: \mathfrak{X}(E) \rightarrow \mathfrak{X}\left(E^{\prime}\right)$ be a Lie algebra isomorphism defined by $\sigma_{*}(X)$ $(f)(e)=X(f \circ \sigma)\left(\sigma^{-1}(e)\right)$ for $X \in \mathfrak{X}(E), f \in C^{\infty}\left(E^{\prime}\right), e \in E^{\prime}$.

\section{§ 2. Preliminaries}

Let $\Gamma$ be a finite group and let $\Gamma \times \tilde{U} \rightarrow \tilde{U}$ be a smooth action on an open set $\tilde{U}$ of $R^{n}$. Let $U=\tilde{U} / \Gamma$ be the orbit space of $\tilde{U}$ and $\pi: \tilde{U} \rightarrow U$ the natural projection. In this section, we shall consider that $p: E \rightarrow U$ is a smooth fibration with generic fibre $F$, a closed connected smooth $m$-manifold, and $E=\tilde{U} \times F / \Gamma$. Let $\mathfrak{X}_{\Gamma}(\tilde{U})$ be the Lie algebra of all $\Gamma$-invariant smooth vector fields on $\tilde{U}$ with compact support. Let $\tilde{E}=\tilde{U} \times F$. Let $\pi: \tilde{E} \rightarrow E$ and $\tilde{p}: \tilde{E} \rightarrow \tilde{U}$ be the natural projections.

Lemma 2. 1. The induced map $\tilde{\pi}_{*}: \mathfrak{X}_{\Gamma}(\tilde{U}) \rightarrow \mathfrak{X}(U)$ is a Lie algebra isomorphism.

Proof. By Bierstone [2] and Schwarz [8], $\tilde{\pi}_{*}$ is epimorphic. For $a \in \tilde{U}$, the isotropy subgroup $\Gamma_{a}$ at a acts on the tangent space $\tau_{a}(\tilde{U})$. Let $\tau_{a}(\tilde{U})^{\Gamma_{a}}$ denote the set of $\Gamma_{a}$-invaraint vectors of $\tau_{a}(\tilde{U})$. Let $X \in \mathfrak{X}_{\Gamma}(\tilde{U})$ such that $\tilde{\pi}_{*}(X)=0$. Then $X_{a} \in \tau_{a}(\tilde{U})^{\Gamma_{a}}$ for any $a \in \tilde{U}$. Since $(d \pi)_{a}: \tau_{a}(\tilde{U}) \rightarrow \tau_{a}(U)$ is monomorphic on $\tau_{a}(\tilde{U})^{\Gamma_{a}}$, we have $X_{a}=0$, and this completes the proof of Lemma 2.1 .

Let $\mathfrak{X}_{\Gamma}(\tilde{E} ; \tilde{p})=\left\{X \in \mathfrak{X}_{\Gamma}(\tilde{E}) ; X\right.$ is fibration preserving $\}$.

Lemma 2.2. The induced map $\tilde{\pi}_{*}: \mathfrak{X}_{\Gamma}(\tilde{E} ; \tilde{p}) \rightarrow \mathfrak{X}(E ; p)$ is a Lie algebra isomorphism.

Proof. By Lemma 2. 1, the map $\tilde{\pi}_{*}$ is monomorphic. Let $X \in \mathfrak{X}$ $(E ; p)$. By Lemma 2.1, there exists a vector field $Y \in \mathfrak{X}_{\Gamma}(\tilde{E})$ such that $\tilde{\pi}_{*}(Y)=X$. We shall prove that $Y$ is fibration preserving. Let $\tilde{e}_{1}, \tilde{e}_{2} \in \tilde{E}$ such that $\tilde{p}\left(\tilde{e}_{1}\right)=\tilde{p}\left(\tilde{e}_{2}\right)=\tilde{b} \in \tilde{U}$. Let $b=\pi(\tilde{b})$ and $e_{i}=\tilde{\pi}\left(\tilde{e}_{i}\right)$ for $i=1,2$. Then $(d \pi)_{\tilde{b}}\left((d \tilde{p})_{\tilde{e}_{i}}\left(Y_{\tilde{e}_{i}}\right)\right)=(d p)_{e_{i}}\left(X_{e_{i}}\right)$ for $i=1,2$. Since $X$ is 
fibration preserving, $(d \pi)_{\tilde{b}}\left((d \tilde{p})_{\tilde{e}_{1}}\left(Y_{\bar{e}_{1}}\right)\right)=(d \pi)_{\tilde{b}}\left((d \tilde{p})_{\tilde{e}_{2}}\left(Y_{\tilde{e}_{2}}\right)\right)$. Note that, if $\Gamma_{\tilde{b}}$ is a principal isotopy group, then $(d \pi)_{\tilde{b}}: \tau_{\tilde{b}}(\tilde{U})^{\Gamma_{\tilde{b}}} \rightarrow \tau_{b}(U)$ is isomorphic, and $(d \tilde{p})_{\tilde{e}_{1}}\left(Y_{\bar{e}_{1}}\right)=(d \tilde{p})_{\tilde{e}_{2}}\left(Y_{\tilde{e}_{2}}\right)$. Since the set of principal orbits of $\tilde{U}$ is open dense in $\tilde{U}$, we see that $Y$ is fibration preserving. This completes the proof of Lemma 2.2.

For $\tilde{e} \in \tilde{E}$, let $\left(V ; x_{1}, \ldots, x_{n}, y_{1}, \ldots, y_{m}\right)$ be a local coordinate at $\tilde{e}$ such that $\left(x_{1}, \ldots, x_{n}\right)$ is a local coordinate of $R^{n}$ and $\left(y_{1}, \ldots, y_{m}\right)$ is a local coordinate of the fibre $F$. We can assume that $V$ is a $\Gamma_{\bar{e}^{-}}$ invariant neighborhood of $\tilde{e}$. Then we see that each $X \in \mathfrak{x}_{\Gamma}(\tilde{E} ; \tilde{p})$ is described on $V$ as follows;

$$
\begin{aligned}
X=\sum_{i=1}^{n} & a_{i}\left(x_{1}, \ldots, x_{n}\right) \frac{\partial}{\partial x_{i}} \\
& +\sum_{j=1}^{m} b_{j}\left(x_{1}, \ldots, x_{n}, y_{1}, \ldots, y_{m}\right) \frac{\partial}{\partial y_{j}},
\end{aligned}
$$

where $a_{i}$ and $b_{j}$ are smooth functions on $V$ 。

Lemma 2.3. If $X \in \mathfrak{X}_{\Gamma}(\tilde{E} ; \tilde{p})$ satisfies $(d \tilde{p})_{\bar{e}} X_{\bar{e}} \neq 0$, then there exists a local coordinate $\left(W ; x_{1}, \ldots, x_{n}, y_{1}, \ldots, y_{m}\right)$ at $\tilde{e}$ such that

(1) $\left(x_{1}, \ldots, x_{n}\right)$ is a local coordinate of $\tilde{U}$ satisfying $x_{i}\left(\tilde{e}_{1}\right)=x_{i}\left(\tilde{e}_{2}\right)$ for $\tilde{b} \in \tilde{U}$ and $\tilde{e}_{1}, \tilde{e}_{2} \in p^{-1}(\tilde{b}) \cap W$.

(2) $x_{1}$ is a $\Gamma_{\tilde{e}}$-invariant smooth function on $W$ such that $X=\frac{\partial}{\partial x_{1}}$.

(3) $\left(y_{1}, \ldots, y_{m}\right)$ is a local coordinate of the fibre $F$.

Proof. We can prove by easy computations.

Lemma 2.4. Let $X \in \mathfrak{X}_{\Gamma}(\tilde{E} ; \tilde{p})$ satisfying $(d \tilde{p})_{\tilde{e}} X_{\bar{\varepsilon}} \neq 0$. Then for any $Y \in \mathfrak{X}_{\Gamma}(\tilde{E} ; \tilde{p})$ with $\operatorname{supp}(Y) \subset W$, there are a neighborhood $W_{1} \subset W$ of $\tilde{e}$ and a vector field $Z \in \mathfrak{X}_{\Gamma}(\tilde{E} ; \tilde{p})$ such that $[X, Z]=Y$ on $W_{1}$, where $W$ is a neighborhood of $\tilde{e}$ as in Lemma 2.3.

Proof. Let $\left(W ; x_{1}, \ldots, x_{n}, y_{1}, \ldots, y_{m}\right)$ be a local coordinate at $\tilde{e}$ as Lemma 2.3. Let $Y=\sum_{i=1}^{n} a_{i}\left(x_{1}, \ldots, x_{n}\right) \frac{\partial}{\partial x_{i}}+\sum_{j=1}^{m} b_{j}\left(x_{1}, \ldots, x_{n}, y_{1}, \ldots, y_{m}\right)$ $\frac{\partial}{\partial y_{j}}$ on W. Put $Z_{1}=\sum_{i=1}^{n}\left(\int_{-\infty}^{x_{1}} a_{i}\left(x_{1}, \ldots, x_{n}\right) d x_{1}\right) \frac{\partial}{\partial x_{i}}+\sum_{j=1}^{m}\left(\int_{-\infty}^{x_{1}} b_{j}\left(x_{1}, \ldots, x_{n}, y_{1}\right.\right.$, $\left.\left.\ldots, y_{m}\right) d x_{1}\right) \frac{\partial}{\partial y_{j}}$. Then $Z_{1}$ is a fibration preserving $\Gamma_{\bar{e}}$-invariant smooth 
vector field on $W$. By using a $\Gamma$-invariant partition of unity on $\tilde{E}$, we can extend $Z_{1}$ to a $\Gamma$-invariant smooth vector field on $\tilde{E}$ with compact support such that $Z=Z_{1}$ on a neighborhood $W_{1} \subset W$ of $\tilde{e}$. Obviously, $[X, Z]=Y$ on $W_{1}$, and this completes the proof of Lemma 2.4 .

Lemma 2.5. (cf. Omori [6], 10.7.1) If $X$ satisfies $X_{\bar{e}} \neq 0$ and $(d p)_{\tilde{e}} X_{\tilde{e}}=0$, then there exists a local coordinate $\left(W ; x_{1}, \ldots, x_{n}, y_{1}, \ldots, y_{m}\right)$ such that $\left(y_{1}, \ldots, y_{m}\right)$ is a local coordinate of the fibre $F$ and

$$
X=\sum_{i=1}^{n} a_{i}\left(x_{1}, \ldots, x_{n}\right) \frac{\partial}{\partial x_{i}}+\frac{\partial}{\partial y_{1}} \text { with } a_{i}(0, \ldots, 0)=0,
$$

where the origin of the coordinate corresponds to the point $\tilde{e}$. Moreover, the coordinate can be chosen such that $y_{1}$ is $\Gamma_{\bar{e}}$-invariant.

Poof. We can show by easy computations.

\section{§ 3. Maximal Ideals of $\mathfrak{X}(B)$ and $\mathfrak{X}(E ; p)$}

Let $B$ be a connected smooth orbifold. Let $p: E \rightarrow B$ be a fibration over $B$ with generic fibre $F$, a connected closed manifold. In this section we shall determine the maximal ideals of $\mathfrak{X}(E ; p)$.

Let $\mathbb{S}_{b}(B)=\{X \in \mathfrak{X}(B) ; X=0$ on a neighborhood of $b$ for $b \in B\}$.

Lemma 3.1 (cf. Koriyama, Maeda, Omori [4], Lemma 4.3) Let $\overline{\mathfrak{M}}$ be a maximal ideal of $\mathfrak{X}(B)$ such that $\overline{\mathfrak{M}} \not \supset[\mathfrak{X}(B), \mathfrak{X}(B)]$. Then there exists a unique point $b \in B$ such that $\overline{\mathfrak{M}} \supset \mathbb{S}_{b}(B)$.

Proof. We give a metric on $B$. Let $\mathscr{S}_{b}(\varepsilon)=\{X \in \mathfrak{X}(B) ; X=0$ on an $\varepsilon$-neighborhood of $b\}$. Assume that $\overline{\mathfrak{M}}+\mathbb{S}_{b}(\varepsilon)=\mathfrak{X}(B)$ for any $b \in B$. As in the proof of Koriyama, Maeda and Omori [4], Lemma 4.1, we can prove that $\overline{\mathfrak{M}} \supset[\mathfrak{X}(B), \mathfrak{X}(B)]$, contradicting the assumption for $\overline{\mathfrak{M}}$. Thus there exists a point $b \in B$ with $\overline{\mathfrak{M}} \supset \mathbb{S}_{b}(\varepsilon)$.

Let $A_{\varepsilon}=\left\{b \in B ; \mathbb{S}_{b}(\varepsilon) \subset \overline{\mathfrak{M}}\right\}$. It is easy to see that, if $\varepsilon>\delta$, then $A_{\varepsilon} \supset c l\left(A_{\delta}\right)$ (the closure of $A$ ). Then there exists $b \in \underset{\varepsilon>0}{\cap} A_{\mathcal{E}}$, and we see that $\overline{\mathfrak{M}} \supset \mathbb{S}_{b}(B)$. If $b \neq b^{\prime}$, then we can prove that $\mathbb{S}_{b}(B)+\mathbb{S}_{b^{\prime}}(B)$ $=\mathfrak{X}(B)$. Therefore such a point $b$ must be unique and this completes the proof of Lemma 3.1. 
Let $B_{0}=\left\{b \in B ; X_{b}=0\right.$ for any $\left.X \in \mathfrak{X}(B)\right\}$ and let $B_{1}=B-B_{0}$. From [1], Lemma 3.9 and Proposition 3.10 we have the following.

Proposition 3.2. (1) For any point $b \in B_{1}$, there exists a unique maximal ideal $\overline{\mathfrak{\Im}}(b)$ such that $\overline{\mathfrak{\Im}}(b) \supset \mathbb{S}_{b}(B), \bar{\Im}(b) \not \supset[\mathfrak{X}(B), \mathfrak{X}(B)]$ and codim $\overline{\mathfrak{\Im}}(b)=\infty$.

(2) Let $\overline{\mathfrak{M}}$ be a maximal ideal of $\mathfrak{X}(B)$ such that $\overline{\mathfrak{M}} \supset \mathbb{S}_{b}(B)$ for $b \in B_{0}$. Then codim $\overline{\mathrm{M}}<\infty$.

Let $B^{*}$ be the space of all maximal ideals $\overline{\mathfrak{M}}$ of $\mathfrak{X}(B)$ such that $\overline{\mathfrak{M}} \not \supset[\mathfrak{X}(B), \mathfrak{X}(B)]$, with Stone topology (see [1], Definition 4。1). Using Lemma 3.1, we define a map $\bar{\tau}: B^{*} \rightarrow B$ such that $\bar{\tau}(\overline{\mathfrak{M}})=b$ if $\overline{\mathfrak{M}} \supset \mathbb{S}_{b}(B)$. Let $B_{1}^{*}=\left\{\overline{\mathfrak{M}} \in B^{*} ; \operatorname{codim} \overline{\mathfrak{M}}=\infty\right\}$.

From [1], Lemma 4.2 we have the following.

Proposition 3. 3. $\quad \bar{\tau}: B_{1}^{*} \rightarrow B_{1}$ is homeomorphic.

Let $\mathbb{S}_{e}(E ; p)=\{X \in \mathfrak{X}(E ; p) ; X=0$ on a neighborhood of $e$ for $e \in E\}$. Then we have the following useful lemma.

Lemma 3.4. Let $\mathfrak{A}$ be a subalgebra of $\mathfrak{X}(E ; p)$ such that $f X \in \mathfrak{X}$ for any $f \in C^{\infty}(E)$ and $X \in \mathfrak{U} \cap\left(\operatorname{Ker} p_{*}\right)$. Let $\mathfrak{M}$ be a maximal ideal of $\mathfrak{A}$ such that $\mathfrak{M} \not \supset[\mathfrak{X}, \mathfrak{R}]$ and $p_{*}(\mathfrak{M})=p_{*}(\mathfrak{Q})$. Then there exists a unique point $e \in E$ such that $\mathfrak{M} \supset \mathbb{S}_{e}(\mathfrak{H})$, where $\mathbb{S}_{e}(\mathfrak{H})=\mathbb{S}_{e}(E ; p) \cap \mathfrak{A}$.

Proof. Suppose that $\mathfrak{A}=\mathfrak{M}+\mathbb{H}_{e}(\varepsilon ; \mathfrak{A})$ for any $e \in E$, where $\mathbb{S}_{e}(\varepsilon ; \mathfrak{A})$ $=\{X \in \mathfrak{A} ; X=0$ on an $\varepsilon$-neighborhood of $e\}$. For any vector field $X \in \mathfrak{A}$, there exists a vector field $Y \in \mathfrak{M}$ such that $Z=X-Y \in \operatorname{Ker} p_{*}$. For each positive number $\varepsilon>0, Z$ can be written as finite sums $Z=\sum Z_{i}$ such that $Z_{i} \in \mathfrak{Y} \cap\left(\operatorname{Ker} p_{*}\right)$ and $\operatorname{diam}\left(\operatorname{supp} Z_{i}\right)<\varepsilon_{0}$. Then as in the proof of Koriyama, Maede and Omori [4], Lemma 4.1, [थ्Y $\cap\left(\operatorname{Ker} p_{*}\right)$, $\left.\mathfrak{U} \cap\left(\operatorname{Ker} p_{*}\right)\right]$ is contained in $\mathfrak{M}$. Then $[\mathfrak{A}, \mathfrak{R}]$ is contained in $\mathfrak{M}$, contradicting the assumption for $\mathfrak{M}$. Therefore there exists a point $e \in E$ such that $\mathfrak{M} \supset \mathbb{S}_{e}(\varepsilon ; \mathfrak{V})$. As in the proof of Lemma 3.1, there exists a unique point $e \in E$ such that $\mathfrak{M} \supset \mathbb{B}_{e}(\varepsilon ; \mathfrak{R})$, and Lemma 3.4 follows. 
Let $F_{b}=p^{-1}(b)$ for $b \in B_{0}$. Put $F_{b, 0}=\left\{e \in F_{b} ; X_{e}=0\right.$ for any $X \in \mathfrak{X}$ $(E ; p)\}$ and put $F_{b, 1}=F_{b}-F_{b, 0}$. Let $\mathfrak{X}_{F}(E ; p)=\left\{X \in \mathfrak{X}(E ; p) ; p_{*}(X)=0\right\}$. For $b \in B_{0}, e \in F_{b}$, put $\mathfrak{\Im}(e)=\left\{X \in \mathfrak{X}(E ; p) ;\left(\left(a d Y_{1}\right) \ldots\left(a d Y_{k}\right) X\right)_{e}=0\right.$ for any $Y_{i} \in \mathfrak{X}_{F}(E ; p)$ and any integer $\left.k \geqq 0\right\}$, where $(\operatorname{ad} Y)(Z)=[Y, Z]$ for $Y, Z \in \mathfrak{X}(E ; p)$.

Lemma 3.5. If $b \in B_{0}$ and $\mathrm{e} \in F_{b, 1}$, then $\mathfrak{S}(e)$ is an infinite codimensional maximal ideal of $\mathfrak{X}(E ; p)$. Moreover $\mathfrak{\Im}(e)$ is a unique maximal ideal containing $\mathbb{S}_{e}(E ; p)$, and $\mathfrak{\Im}(e) \not \supset\left[\mathfrak{X}_{F}(E ; p), \mathfrak{X}_{F}(E ; p)\right]$.

Proof. There exist a finite group $\Gamma$, a smooth action of $\Gamma$ on an open set $\tilde{U}$ of $R^{n}$ such that $\tilde{U} / \Gamma=U$ is a neighborhood of $b$, and there exists a smooth $\Gamma$-action on the fibre $F$ such that $p^{-1}(U)=$ $\tilde{U} \times F / \Gamma$. Put $E_{U}=\tilde{U} \times F / \Gamma$ and $\tilde{E}_{U}=\tilde{U} \times F$. Let $\tilde{\pi}: \tilde{E}_{U} \rightarrow E_{U}$ and $\tilde{p}: \tilde{E}_{U}$ $\rightarrow \tilde{U}$ be the projections. By Lemma $2.2 \tilde{\pi}_{*}: \mathfrak{X}_{\Gamma}\left(\tilde{E}_{U} ; \tilde{p}\right) \rightarrow \mathfrak{X}\left(E_{U} ; p\right)$ is a Lie algebra isomorphism. Let $\tilde{e}$ be a point of $\tilde{E}_{U}$ such that $\pi(\tilde{e})=e$, and let $\tilde{\Im}(\tilde{e})=\left\{X \in \mathfrak{X}_{\Gamma}\left(\tilde{E}_{U} ; \tilde{p}\right) ;\left(\left(a d Y_{1}\right) \ldots\left(\text { ad } Y_{k}\right) X\right)_{\tilde{e}}=0\right.$ for any $Y_{i} \in \mathfrak{X}_{\Gamma . F}$ $\left(\tilde{E}_{U} ; \tilde{p}\right)$ and any integer $\left.k \geqq 0\right\}$. Note that $\mathfrak{X}_{\Gamma}\left(\tilde{E}_{U} ; \tilde{p}\right)$ can be identified with $\mathfrak{X}_{\Gamma}(\tilde{U}) \times \mathfrak{X}_{\Gamma, F}\left(\tilde{E}_{U} ; \tilde{p}\right)$ as an $R$-algebra, where $\mathfrak{X}_{\Gamma, F}\left(\tilde{E}_{U} ; \tilde{p}\right)=\left\{X \in \mathfrak{X}_{\Gamma}\right.$ $\left.\left(\tilde{E}_{U} ; \tilde{p}\right) ; \tilde{p}_{*} X=0\right\}$. By easy computations we can see that $\tilde{\Im}(\tilde{e})$ is an ideal of $\mathfrak{X}_{\Gamma}\left(\tilde{E}_{U} ; \tilde{p}\right)$. Since $\tilde{\pi}_{*}^{-1}\left(\widetilde{S}(e) \cap \mathfrak{X}\left(E_{U} ; p\right)\right)=\tilde{\Im}(\tilde{e}), \mathfrak{S}(e)$ is an ideal of $\mathfrak{X}(E ; \mathrm{p})$.

Let $\mathfrak{M}$ be a maximal ideal containing $\mathbb{S}_{e}(E ; p)$. Assume that there exists a vector field $X \in \mathfrak{M}$ with $X_{e} \neq 0$. There exists $\tilde{X} \in \mathfrak{X}_{\Gamma}\left(\tilde{E}_{U} ; \tilde{p}\right)$ such that $\pi_{*}(\tilde{X})=X$ on a neighborhood $V$ of $e$. Since $b \in B_{0},(d \tilde{p})_{\tilde{e}} \tilde{X}_{\bar{e}}$ $=0$. By Lemma 2.5 , there exists a local coordinate $\left(W ; x_{1}, \ldots, x_{n}\right.$, $\left.y_{1}, \ldots, y_{m}\right)$ around $e$ such that $\left(y_{1}, \ldots, y_{m}\right)$ is a local coordinate for a fibre $F$ and $\tilde{X}=\sum_{i=1}^{n} a_{i}\left(x_{1}, \ldots, x_{n}\right) \frac{\partial}{\partial x_{i}}+\frac{\partial}{\partial y_{1}}$ with $a_{i}(0, \ldots, 0)=0$, where the origin of the coordinate corresponds to the point $\tilde{e}$. There is a vector fields $\tilde{X}_{1} \in \mathfrak{X}_{\Gamma, F}\left(\tilde{E}_{U} ; \tilde{p}\right)$ such that $\tilde{X}_{1}=y_{1} \frac{\partial}{\partial y_{1}}$ on a neighborhood $W_{1} \subset W$ or $\tilde{e} . \quad$ Note that $\tilde{X}_{2}=\left[\tilde{X}, \tilde{X}_{1}\right]=\frac{\partial}{\partial y_{1}}$ on $W_{1}$. Put $X_{2}=\left[X, \tilde{\pi}_{*}\right.$ $\left.\left(\tilde{X}_{1}\right)\right]$. Then $X_{2} \in \mathfrak{M}$. Let $Y \in \mathfrak{X}\left(E_{U} ; p\right)$ such that $\sup p(Y) \subset V$. Let $\tilde{Y} \in \mathfrak{X}_{\Gamma}\left(\tilde{E}_{U} ; \tilde{p}\right)$ such that $\tilde{\pi}_{*}(\tilde{Y})=Y$. As in the proof of Lemma 2.4, we can prove that there are a neighborhood $W_{1}$ of $e$ and $a$ vector field $\tilde{Z} \in \mathfrak{X}_{\Gamma}\left(\tilde{E}_{U} ; \tilde{p}\right)$ such that $\left[\tilde{X}_{2}, \tilde{Z}\right]=\tilde{Y}$ on $W_{1} \subset W$. Put $Z=\tilde{\pi}_{*}(\tilde{Z})$. 
Then $\left[X_{2}, Z\right] \in \mathfrak{M}$ and $\left[X_{2}, Z\right]=Y$ on a neighborhood of $e$. Since $\mathfrak{M}$ contains $\mathbb{S}_{e}(E ; p), \mathfrak{M}=\mathfrak{X}(E ; p)$. This is a contradiction. Thus $X_{e}=0$ for any $X \in \mathfrak{M}$, and we have $\mathfrak{M}=\mathfrak{S}(e)$. Therefore $\mathfrak{\Im}(e)$ is a unique maximal ideal containing $\mathcal{S}_{e}(E ; p)$. There are vector fields $\tilde{Y}_{i} \in \mathfrak{X}_{\Gamma}$ $\left(\tilde{E}_{U} ; \tilde{p}\right)(i=0,1,2, \ldots)$ such that $\tilde{Y}_{i}=y_{1}^{i} \frac{\partial}{\partial y_{1}}$ on a neighborhood of $\tilde{e}$. Put $Y_{i}=\tilde{\pi}_{*}\left(\tilde{Y}_{i}\right)$. Then $Y_{i} \notin \widetilde{\Im}(e)$, and $\widetilde{\mathfrak{S}}(e)$ is infinite codimensional. Since $\left[Y_{0}, Y_{1}\right] \notin \mathfrak{S}(e), \mathfrak{S}(e) \not \supset\left[\mathfrak{X}_{F}(E ; p), \mathfrak{X}_{F}(E ; p)\right]$. This completes the proof of Lemma 3.5.

Let $b \in B_{0}$ and $e \in F_{b, 0}$. Let $U=\tilde{U} / \Gamma$ be a neighborhood of $b$ as in $\S 2$. For $X \in \mathfrak{X}(E ; p)$, there is $Y \in \mathfrak{X}_{\Gamma}(\tilde{U} \times F ; \tilde{p})$ such that $\tilde{\pi}_{*}(Y)=X$ on a neighborhood $W$ of $e$, where $\tilde{\pi}: \tilde{U} \times F \rightarrow \tilde{U} \times F / \Gamma$ is the natural projection.

Choose a point $\tilde{e} \in \tilde{U} \times F$ with $\tilde{\pi}(\tilde{e})=e$. Let $j_{\tilde{e}}^{1}(Y)$ denote the 1 -jet of $Y$ at $\tilde{e}$. Note that $j_{\tilde{\varepsilon}}^{1}(Y)$ defines an element of $\operatorname{gl}_{\Gamma_{\tilde{e}}}\left(\tau_{\tilde{e}}(\tilde{U} \times F)\right)$, where $\mathrm{gl}_{\Gamma_{\tilde{e}}}\left(\tau_{\tilde{\varepsilon}}(\tilde{U} \times F)\right)$ denotes the set of all $\Gamma_{\tilde{e}}$-invariant endmorphism of the tangent space $\tau_{\tilde{e}}((\tilde{U} \times F))$ at $\tilde{e} . \quad$ Let $J_{e}^{1}(E ; p)=\left\{\mathrm{A} \in \mathfrak{g l}_{\Gamma_{\tilde{e}}}\left(\tau_{\tilde{e}}(\tilde{U} \times F)\right)\right.$; $(d \tilde{p})_{\tilde{e}}(\mathrm{~A}(v))=0$ for any $v \in \tau_{\tilde{e}}(\tilde{U} \times F)$ with $\left.(d \tilde{p})_{\tilde{\varepsilon}}(v)=0\right\}$, where $\tilde{p}: \tilde{U} \times$ $F \rightarrow \tilde{U}$ is the natural projection. Since $Y$ is fibration preserving, $j_{\tilde{E}}^{1}(Y)$ $\in J_{e}^{1}(E ; p)$. Let $j_{e}^{1}: \mathfrak{X}(E ; p) \rightarrow J_{e}^{1}(E ; p)$ be a map defined by $j_{e}^{1}(X)=j_{\tilde{e}}^{1}(Y)$. Then it is easy to see the following.

Lemma 3. 6. $j_{e}^{1}: \mathfrak{X}(E ; p) \rightarrow J_{e}^{1}(E ; p)$ is an onto Lie algebra homomorphism.

Lemma 3.7. Let $\mathfrak{M}$ be a maximal ideal of $\mathfrak{X}(E ; p)$ such that $p_{*}(\mathfrak{M})$ $=\mathfrak{X}(B)$ and $\mathfrak{M} \supset \mathbb{S}_{e}(E ; p)$ for some point $e \in F_{b, 0}$ with $b \in B_{0}$. Then $\mathfrak{M}=\left(j_{e}^{1}\right)^{-1}(\mathfrak{Q})$ for some maximal ideal $\mathfrak{Q}$ of $J_{e}^{1}(E ; p)$, and $\mathfrak{M}$ is finite codimensional.

Proof. Assume that $j_{e}^{1}(\mathfrak{M})=J_{e}^{1}(E ; p)$. We use the same notations as in the proof of Lemma 3.8. Take a vector field $X \in \mathfrak{M}$ such that $j_{e}^{1}(X)$ is a unit matrix. There exists $Y \in \mathfrak{X}_{\Gamma}(\tilde{U} \times F ; \tilde{p})$ such that $\tilde{\pi}_{*}(Y)=X$ on a neighborhood of $e$. By Sternberg's linearization theorem [9], there exists a local coordinate $\left(W ; z_{1}, \ldots, z_{n+m}\right)$ at $e$ such that $Y=\sum_{i=1}^{n+m} z_{i} \frac{\partial}{\partial z_{i}}$ on $W$. 
Let $Z \in \mathfrak{X}(E ; p)$ with $j_{e}^{1}(Z)=0$. There exists $\tilde{Z} \in \mathfrak{X}_{\Gamma}(\tilde{U} \times F ; \tilde{p})$ such that $\tilde{\pi}_{*}(\tilde{Z})=Z$ on a neighborhood of $e$. Then $j_{\tilde{e}}^{1}(\tilde{Z})=0$. Let $\tilde{Z}_{1}=\int_{0}^{\infty} A d$ $(\exp t Y) \tilde{Z} d t$, where $(\operatorname{Ad}(\exp t Y) \tilde{Z})_{x}=\left(d \phi_{t}\right)_{\phi_{t}^{-1}(x)} \tilde{Z}_{\phi_{t}^{-1}(x)}, \phi_{t}=\exp t Y$ (see Koriyama, Maeda and Omori [5], § 1). $\quad \tilde{Z}$ can be written in the form $\tilde{Z}=\sum_{i=1}^{n+m} a_{i}\left(z_{1}, \ldots, z_{n+m}\right) \frac{\partial}{\partial z_{i}}$ on $W$ with $\frac{\partial a_{i}}{\partial z_{j}}(0, \ldots, 0)=0$ for $i, j=1, \ldots$, $n+m$. Then $\operatorname{Ad}(\exp t Y) \tilde{Z}=\sum_{i=1}^{n+m} a_{i}\left(e^{-t} z_{1}, \ldots, e^{-t} z_{n+m}\right) e^{t} \frac{\partial}{\partial z_{i}}$ on $W$. Since $\frac{\partial a_{i}}{\partial z_{j}}(0, \ldots, 0)=0$, it is clear that $\int_{0}^{\infty} a_{i}\left(e^{-t} z_{1}, \ldots, e^{-t} z_{n+m}\right) e^{t} d t$ exists. Hence $\tilde{Z}_{1} \in \mathfrak{X}_{\Gamma}(\tilde{U} \times F)$. We can see that $\exp s(\operatorname{Ad}(\exp t Y) \tilde{Z})=\phi_{t} \circ \phi_{s}^{\circ} \phi_{t}^{-1}$, where $\phi_{s}=\exp s \tilde{Z}$. Thus $\tilde{Z}_{1}$ is fibration preserving, and $\tilde{Z}_{1} \in \mathfrak{X}_{\Gamma}(\tilde{U} \times F ; \tilde{p})$. Note that $\left[Y, \tilde{Z}_{1}\right]=\tilde{Z}$, and $\left[X, \tilde{\pi}_{*}\left(\tilde{Z}_{1}\right)\right]=Z$ on a neighborhood of $e$. Since $\mathfrak{M}$ contains $\mathfrak{S}_{e}(E ; p)$, $\mathfrak{M}$ contains the ideal $\operatorname{Ker} j_{e}^{1}$ of $\mathfrak{X}(E ; p)$. But $j_{e}^{1}(\mathfrak{M})=J_{e}^{1}(E ; p)$, hence $\mathfrak{M}=\mathfrak{X}(E ; p)$. This is a contradiction. Thus $j_{e}^{1}(\mathfrak{M})$ must be a proper ideal $\mathfrak{L}$ of $J_{e}^{1}(E ; p)$, and $\mathfrak{M}=\left(j_{e}^{1}\right)^{-1}(\mathfrak{L})$. Since $J_{e}^{1}(E ; p)$ is a finite dimensional Lie algebra, $\mathfrak{M}$ is finite codimensional. This completes the proof of Lemma 3.7.

Let $E^{*}$ be the space of all maximal ideals $\mathfrak{M}$ of $\mathfrak{X}(E ; p)$ such that $\mathfrak{M} \not \supset[X(E ; p), \mathfrak{X}(E ; p)]$, with Stone topology. Let $E_{1}^{*}=\left\{\mathfrak{M} \in E^{*}\right.$; codim $\mathfrak{M}=\infty$. Combining Proposition 3.2, Lemmas 3.4, 3.5 and 3.7, we have the following.

Proposition 3. 8. If $\mathfrak{M} \in E_{1}^{*}$, then

(1) $\mathfrak{M}=p_{*}^{-1}(\overline{\mathfrak{M}})$ for some $\overline{\mathfrak{M}} \in B_{1}^{*}$, or

(2) $\mathfrak{M}=\mathfrak{S}(e)$ for a point $e \in F_{b, 1}$ with $b \in B_{0}$.

Let $E_{1}=B_{1} \cup\left(\cup_{b \in B_{0}} F_{b, 1}\right)$ be a subspace of the disjoint union $B \cup E$. Using Proposition 3.8 we can define a map $\tau: E_{1}^{*} \rightarrow E_{1}$ as follows.

(1) If $\mathfrak{M}=p_{*}^{-1}(\overline{\mathfrak{M}})$ for some $\overline{\mathfrak{M}} \in B_{1}^{*}$, then $\tau(\mathfrak{M})=\bar{\tau}(\overline{\mathfrak{M}})$.

(2) If $\mathfrak{M}=\mathfrak{S}(e)$ for $e \in F_{b, 1}$ with $b \in B_{0}$, then $\tau(\mathfrak{M})=e$.

Proposition 3. 9. $\tau: E_{1}^{*} \rightarrow E_{1}$ is homeomorphic.

Proof. By Proposition 3.8, $\tau$ is a bijection. It is enough to show that $\tau(C L(S))=c l(\tau(S))$ for any subset $S$ of $E_{1}^{*}$. Here $C L$ and $c l$ are closure operators of $E_{1}^{*}$ and $E_{1}$, respectively. Assume that 
$\tau(\mathfrak{M}) \notin \operatorname{cl}(\tau(S))$ for some $\mathfrak{M} \in C L(S)$. In the case that $\tau(\mathfrak{M})=\mathrm{b} \in B_{1}$, we can find $X \in \mathfrak{X}(E ; p)$ such that $p_{*}(X)_{b} \neq 0$ and $X=0$ on a neighborhood of $c l(\tau(S))$. Then we see that $X \in \cap_{\mathfrak{x} \in S} \mathfrak{R} \subset \mathbb{M}$ (cf. [1], $\S 4)$. Since $p_{*}(\mathfrak{M})=\mathfrak{I}(b), p_{*}(X)_{b}=0$. This is a contradiction. In the case that $\tau(\mathfrak{M})=e \in F_{b, 1}\left(b \in B_{0}\right)$, we can find $X \in \mathfrak{X}(E ; p)$ such that $p_{*}(X)=0, \quad X_{e} \neq 0$ and $X=0$ on a neighborgood of $\tau(S)$. Then $X \in \cap \mathfrak{R} \subset \mathfrak{M}=\mathfrak{S}(e)$, and $X_{e}=0$. This is a contradiction. Therefore $\tau(C L(S))$ is contained in $\operatorname{cl}(\tau(S))$.

If $X \in \underset{\mathfrak{M} \in S \cap \tau^{-1}\left(B_{1}\right)}{\cap} \mathbb{M}$, then $p_{*}(X)=0$ on $\tau(S) \cap B_{1}$, so $p_{*}(X)=0$ on $c l\left(\tau(S) \cap B_{1}\right)$. Hence, for any $X \in \underset{\mathfrak{R} \in S}{\cap \mathfrak{M}}, p_{*}(X)=0$ on $\operatorname{cl}\left(\tau(S) \cap B_{1}\right)$. Then $\underset{\mathfrak{R} \in S}{\cap \mathfrak{M}}$ is an ideal of $\mathfrak{X}(E ; p)$ contained in $p_{*}^{-1}(\widetilde{\mathfrak{S}}(b))$ for any $b \in \operatorname{cl}\left(\tau(S) \cap B_{1}\right)$. Similarly, $\underset{\mathfrak{M} \in S}{\cap \mathfrak{M}}$ is contained in $\mathfrak{S}(e)$ for any $e \in c l$ $\left(\tau(S) \cap p^{-1}\left(B_{0}\right)\right)$. Thus $\underset{\mathfrak{R} \in S}{\mathfrak{M}}$ is contained in $\underset{\tau(\mathfrak{R}) \in \operatorname{cl}(\tau(S))}{\cap} \mathfrak{M}$, and $\tau(C L(S))$ contains $c l(\tau(S))$. This completes the proof of Proposition 3.9.

\section{§4. Maximal Ideals of Some Subalgebras of $\mathfrak{X}(\mathbb{E} ; \not)$}

Let $\mathfrak{X}(B)_{b}=\left\{X \in \mathfrak{X}(B) ; X_{b}=0\right\}$ for $b \in B$, and $\mathfrak{A}_{b}=p_{*}^{-1}\left(\mathfrak{X}(B)_{b}\right)$ be a subalgebra of $\mathfrak{X}(E ; p)$. For $b, b^{\prime} \in B$, let $\hat{F}_{b^{\prime}, 0}^{b}=\left\{e \in F_{b^{\prime}} ; X_{e}=0\right.$ for $\left.X \in \mathfrak{A}_{b}\right\}$ and let $\hat{F}_{b^{\prime}, 1}^{b}=F_{b^{\prime}}-\hat{F}_{b^{\prime}, 0}^{b}$. Let $\mathfrak{\Im}(e)_{b}=\mathfrak{U}_{b} \cap \mathfrak{S}(e)$ and let $\mathbb{S}_{e}\left(\mathfrak{U}_{b}\right)$ $=\mathfrak{U}_{b} \cap \mathbb{S}_{e}(E ; p)$ for $e \in E$. As in the proofs of Lemmas 3.5 and 3.7, we prove the following lemmas respectively.

Lemma 4.1. If $e \in \hat{F}_{b^{\prime}, 1}^{b}$ for $b^{\prime} \in B_{0}$ or $b^{\prime}=b, \widetilde{\Im}(e)_{b}$ is an infinite codimensional maximal ideal of $\mathfrak{U}_{b}$. Moreover $\mathfrak{\Im}(e)_{b}$ is a unique maximal ideal containing $\mathfrak{S}_{e}\left(\mathfrak{U}_{b}\right)$.

Lemma 4.2. Let $\mathfrak{M}$ be a maximal ideal of $\mathfrak{U}_{b}$ such that $p_{*}(\mathfrak{M})=$ $\mathfrak{X}(B)_{b}$ and $\mathfrak{M} \supset \mathbb{S}_{e}\left(\mathfrak{A}_{b}\right)$ for some $e \in \hat{F}_{b^{\prime}, 0}^{b}$ with $b^{\prime} \in B_{0}$ or $b^{\prime}=b$. Then $\mathfrak{M}$ is finite codimensional.

Let $A_{b}^{*}$ be the set of all maximal ideals $\mathfrak{M}$ of $\mathfrak{A}_{b}$ such that $\mathfrak{M} \not\left[\mathfrak{U}_{b}, \mathfrak{U}_{b}\right]$ and $p_{*}(\mathfrak{M})=\mathfrak{X}(B)_{b}$. Let $A^{*}=\left\{\mathfrak{M} ; \mathfrak{M} \in A_{b}^{*}\right.$ for some $\left.b \in B\right\}$. We give the Stone topology on $A^{*}$. Combining Lemmas 3.4, 4.1 and 4. 2 we have the following.

Proposition 4.3. If $\mathfrak{M} \in A_{b}^{*}$, then we have the following cases. 
(1) There exists a point $e \in p^{-1}\left(B_{0}\right)$ such that $\mathfrak{M} \supset \mathbb{S}_{e}\left(\mathfrak{U}_{b}\right)$, and $\mathfrak{M}=$ $\hat{\mathfrak{M}} \cap \mathfrak{A}_{b}$ for some $\hat{\mathfrak{M}} \in E^{*}$.

(2) There exists a point $e \in F_{b}\left(b \in B_{1}\right)$ such that $\mathfrak{M} \supset \mathfrak{S}_{e}\left(\mathfrak{H}_{b}\right)$, and $\mathfrak{M} \subset \widehat{\mathfrak{M}}$ for any $\hat{\mathfrak{M}} \in E^{*}$.

Using Lemma 3.4, we have a map $t: A^{*} \rightarrow E$ such that $\mathfrak{M} \supset \mathcal{S}_{t(\mathfrak{R})}\left(\mathfrak{U}_{b}\right)$ for $\mathfrak{M} \in \mathfrak{Y}_{b}^{*}$. Let $\hat{A}_{b, 1}^{*}=\left\{\mathfrak{M} \in A_{b}^{*} ; \operatorname{codim} \mathfrak{M}=\infty\right.$ and $\mathfrak{M} \subset \hat{\mathfrak{M}}$ for any $\left.\hat{\mathfrak{M}} \in E^{*}\right\}$ for $b \in B_{1}$ and $\hat{A}_{b, 1}^{*}=\left\{\mathfrak{M} \in A^{*} ; \operatorname{codim} \mathfrak{M}=\infty, p(t(\mathfrak{M}))=b\right\}$ for $b \in B_{0}$. Let $\hat{A}_{1}^{*}=\cup_{b \in B} A_{b, 1}^{*}$. Setting $\hat{E}_{1}=\cup_{b \in B} \hat{F}_{b, 1}^{b}$, we have a bijective map $t: \hat{A}_{1}^{*} \rightarrow \hat{E}_{1}$ by Lemma 4.1 and Proposition 4.3 .

Proposition 4. 4. The map $t: \hat{A}_{1}^{*} \rightarrow \hat{E}_{1}$ is homeomorphic.

Proof. It is enough to show that $t(C L(S))=c l(t(S))$ for any subset $S$ of $\hat{A}_{1}^{*}$, where $C L$ and $c l$ are closure operators of $\hat{A}_{1}^{*}$ and $\hat{E}_{1}$ respectively. As in the proof of Proposition 3.9, we see that $t(C L(S))$ is contained in $\operatorname{cl}(t(S))$.

Note that, for $X \in \mathfrak{X}(E ; p)$ and $e \in \hat{E}_{1}, \quad X \in \mathfrak{S}(e)_{p(e)}$ if $\left(\left(\operatorname{ad} Y_{1}\right) \ldots\right.$ $\left.\left(a d Y_{k}\right) X\right)_{e}=0$ for any $Y_{i} \in \mathfrak{X}_{F}(E ; p)$ and any integer $k \geqq 0$. Then we see that $\underset{\mathrm{m} \in S}{\cap \mathfrak{M}}=\bigcap_{e \in t(s)} \mathfrak{S}(e)_{p(e)}=\underset{e \in c l(t(s))}{\cap} \mathfrak{S}(e)_{p(e)}$. Thus $c l(t(S))$ is contained in $t(C L(S))$. This completes the proof of Proposition 4. 4.

\section{§5. Theorem 0.2 (2) Implies (3)}

Let $B$ and $B^{\prime}$ be connected smooth orbifolds without boundary. Let $p: E \rightarrow B\left(\right.$ resp. $\left.p^{\prime}: E^{\prime} \rightarrow B^{\prime}\right)$ be a fibration over $B$ (resp. $B^{\prime}$ ) with generic fibre $F$ (resp. $F^{\prime}$ ), a connected closed manifold. Suppose that there exists a Lie algebra isomorphism $\Phi: \mathfrak{X}(E ; p) \rightarrow \mathfrak{X}\left(E^{\prime} ; p^{\prime}\right)$.

Lemma 5.1. $\Phi\left(\mathfrak{X}_{F}(E ; p)\right)=\mathfrak{X}_{F^{\prime}}\left(E^{\prime} ; p^{\prime}\right)$.

Proof. From Proposition 3.9, there exists a homeomorphism $\phi$ : $B_{1} \cup\left(\bigcup_{b \in B_{0}} F_{b, 1}\right) \stackrel{\tau^{-1}}{\longrightarrow} E_{1}^{*} \stackrel{\Phi}{\longrightarrow} E_{1}^{\prime *} \stackrel{\tau^{\prime}}{\longrightarrow} B_{1}^{\prime} \cup\left(\underset{b^{\prime} \in B^{\prime} 0}{\bigcup} F_{b^{\prime}, 1}\right)$. Note that $B_{1}, B_{1}^{\prime}, F_{b, 1}$ and $F_{b^{\prime}, 1}$ are connected smooth orbifolds. Assume that $\phi\left(B_{1}\right)=F_{b^{\prime}, 1}^{\prime}$ for some $b^{\prime} \in B_{0}$. Put $\mathfrak{A}_{b^{\prime}, 0}=\left\{X \in \mathfrak{X}\left(E^{\prime} ; p^{\prime}\right) ; X=0\right.$ on $\left.p^{\prime-1}\left(b^{\prime}\right)\right\}$. We see that $\underset{b \in B_{1}}{\cap} \tau^{-1}(b)=\mathfrak{X}_{F}(E ; p)$ and $\underset{e^{\prime} \in F^{\prime} b^{\prime}, 1}{\cap} \tau^{\prime-1}\left(e^{\prime}\right)=\mathfrak{U}_{b^{\prime}, 0}$. Let $E_{1, F}=\{e \in E$; $X_{e} \neq 0$ for some $\left.X \in \mathfrak{X}_{F}(E ; p)\right\}$. Using the method of the proof of 
[1], Proposition 3.2, we see that $E_{1, F}$ is dense in $E$. As in the proof of Lemma $3.5, \mathfrak{J}(e) \cap \mathfrak{X}_{F}(E ; p)$ is an infinite codimensional maximal ideal of $\mathfrak{X}_{F}(E ; p)$ and we see that $\mathfrak{S}(e) \cap \mathfrak{X}_{F}(E ; p) \perp\left[\mathfrak{X}_{F}(E ; p), \mathfrak{X}_{F}(E ; p)\right]$. Hence the intersection of all maximal ideals $\mathfrak{M}$ of $\mathfrak{X}_{F}(E ; p)$ with $\mathfrak{M} D\left[\mathfrak{X}_{F}(E ; p), \mathfrak{X}_{F}(E ; p)\right]$ is zero.

Let $\mathfrak{M}^{\prime}$ be a maximal ideal of $\mathfrak{Y}_{b^{\prime}, 0}^{\prime}$ with $\mathfrak{M} \not \supset\left[\mathfrak{U}_{b^{\prime}, 0}^{\prime}, \mathfrak{U}_{b^{\prime}, 0}^{\prime}\right]$. If $p_{*}^{\prime}\left(\mathfrak{M}^{\prime}\right)$ is a proper ideal of $\mathfrak{X}\left(B^{\prime}\right)$, then $\mathfrak{M}^{\prime}=\left(p_{*}^{\prime}\right)^{-1}\left(\overline{\mathfrak{M}^{\prime}}\right)$ for some $\overline{\mathfrak{M}^{\prime}} \in B^{\prime *}$ 。 If $\mathrm{p}_{*}^{\prime}\left(\mathfrak{M}^{\prime}\right)=\mathfrak{X}\left(B^{\prime}\right)$, then, by Lemma 3.4 , there exists a unique point $e^{\prime} \in E^{\prime}$ such that $\mathfrak{M}^{\prime} \supset \mathbb{S}_{e^{\prime}}\left(\mathfrak{U}_{b^{\prime}, 0}^{\prime}\right)$ for $e^{\prime} \in p^{\prime-1}\left(B_{0}^{\prime}\right)$ or $p^{\prime}\left(e^{\prime}\right)=b^{\prime}$, where $\mathbb{S}_{e^{\prime}}\left(\mathfrak{U}_{b^{\prime}, 0}^{\prime}\right)=\mathbb{S}_{e^{\prime}}\left(E^{\prime} ; p^{\prime}\right) \cap \mathfrak{Y}_{b^{\prime}, 0}^{\prime}$. Choose a point $e_{1}^{\prime} \in E^{\prime}$ with $p^{\prime}\left(e_{1}^{\prime}\right) \in B_{1}^{\prime}$. There exists $X^{\prime} \in \mathfrak{U}_{b^{\prime}, 0}^{\prime} \cap\left(\operatorname{Ker} p_{*}^{\prime}\right)$ such that $X_{e_{1}^{\prime}}^{\prime} \neq 0$ and $X^{\prime}=0$ on a neighborhood of $p^{\prime-1}\left(B_{0}^{\prime}\right)$. Then $X^{\prime}$ is contained in any maximal ideal of $\mathfrak{U}_{b^{\prime}, 0}^{\prime}$. But, for each maximal ideal $\mathfrak{M}^{\prime}$ of $\mathfrak{U}_{b^{\prime}, 0}^{\prime}$ with $\mathfrak{M}^{\prime} \supset\left[\mathfrak{X}_{b^{\prime}, 0}^{\prime}\right.$ $\left.\mathfrak{Y}_{b^{\prime}, 0}^{\prime}\right]$, there exists a maximal ideal $\mathfrak{M}$ of $\mathfrak{X}_{F}(E ; p)$ with $\mathfrak{M} \not \supset\left[\mathfrak{X}_{F}(E ; p)\right.$, $\mathfrak{X}(E ; \mathrm{p})]$ such that $\Phi(\mathfrak{M})=\mathfrak{M}^{\prime}$. Thus the intersection of these maximal ideals $\mathfrak{M}^{\prime}$ of $\mathfrak{Y}_{b^{\prime}, 0}^{\prime}$ must be zero, and this is a contradiction. Therefore $\phi\left(B_{1}\right)=B_{1}^{\prime}$ and we have $\Phi\left(\mathfrak{X}_{F}(E ; p)\right)=\mathfrak{X}_{F^{\prime}}\left(E^{\prime} ; p^{\prime}\right)$. This completes the proof of Lemma 5.1 .

Proposition 5.2. If there exists a Lie algebra isomorphism $\Phi: \mathfrak{X}(E$; $p) \rightarrow \mathfrak{X}\left(E^{\prime} ; p^{\prime}\right)$, then there exists a Lie algebra isomorphism $\bar{\Phi}: \mathfrak{X}(B) \rightarrow \mathfrak{X}\left(B^{\prime}\right)$ and a diffeomorphism $\bar{\sigma}: B \rightarrow B^{\prime}$ such that $p_{*}^{\prime} \circ \Phi=\bar{\Phi} \circ p_{*}$ and $\bar{\Phi}=\bar{\sigma}_{*}$.

Proof. By Lemma 5.1, the Lie algebra isomorphism $\Phi$ induces a Lie algebra isomorphism $\bar{\Phi}: \mathfrak{X}(B) \rightarrow \mathfrak{X}\left(B^{\prime}\right)$. Using a partition of unity, we can reduce the proof of Proposition 5.2 to the special case when the orbifolds $B$ and $B^{\prime}$ are orbit spaces of representation spaces of finite groups. Thus Proposition 5.2 follows from Theorem of [1].

We shall use the notations given in $\S 4$. From Proposition 5.2, we have a Lie algebra isomorphism $\Phi: \mathfrak{U}_{b} \rightarrow \mathfrak{U}_{\tilde{\sigma}(b)}^{\prime}$ for each $\mathrm{b} \in B$. By Proposition 4.4 , there exists a homeomorphism $\sigma_{1}: \hat{E}_{1} \stackrel{t^{-1}}{\longrightarrow} \hat{A}_{1}^{*} \stackrel{\Phi}{\longrightarrow} \hat{A}_{1}^{*}$ $\stackrel{t^{\prime}}{\longrightarrow} \hat{E}_{1}^{\prime}$

Proposition 5.3. The map $\sigma_{1}: \hat{E}_{1} \rightarrow \hat{E}_{1}^{\prime}$ is extended to a homeomorphism $\sigma: E \rightarrow E^{\prime}$ such that $p^{\prime} \circ \sigma=\bar{\sigma} \circ p$.

Let $\hat{A}_{b}^{*}=\left\{\mathfrak{M} \in A_{b}^{*} ; \mathfrak{M} \not \subset \hat{\mathfrak{M}}\right.$ for any $\left.\hat{\mathfrak{M}} \in E^{*}\right\}$ for $\mathrm{b} \in B_{1}$ and $\hat{A}_{b}^{*}=\{\mathfrak{M}$ 
$\left.\in A^{*} ; p(t(\mathfrak{M}))=b\right\}$ for $b \in B_{0} . \quad$ Let $\hat{A}^{*}=\left\{\mathfrak{M} ; \mathfrak{M} \in \hat{A}_{b}^{*}\right.$ for some $\left.b \in B\right\}$. Let $C L$ and $c l$ be the closure operators of $\hat{A}^{*}$ and $E$, respectively. To prove Proposition 5.3, we need the following.

Lemma 5. 4. $t(C L(S))=\operatorname{cl}(t(S))$ for any subset $S$ of $\hat{A}^{*}$.

Proof. As in the proof of Proposition 3.9, we see that $t(C L(S))$ is contained in $\operatorname{cl}(t(S))$. Let $e \in c l(t(S))$. By the similar argument to Proposition 4.4, we see that $\cap \mathfrak{M}$ is contained in $\mathfrak{S}(e)_{p(e)}$ for $e \in c l(t(S)) \cap \hat{E}_{1}$. Thus $c l(t(S)) \cap \hat{E}_{1}$ is contained in $t(C L(S))$.

Now assume that $j_{e}^{1}(\mathfrak{N})=J_{e}^{1}\left(\mathfrak{U}_{b}\right)$ for some $e \in c l(t(S)) \cap \hat{F}_{b, 0}^{b}$, where $\mathfrak{N}=\bigcap \mathfrak{M} \in S$ M,$b=p(e)$. As in the proof of Lemma 3.7, we can prove that, for any $X \in \mathfrak{U}_{b} \cap\left(\operatorname{Ker} p_{*}\right)$, there exists $Y \in \mathfrak{N}$ such that $X=Y$ on an open neighborhood $W$ of $e$. There exists $\mathfrak{M} \in S$ with $t(\mathfrak{M}) \in W$. Since $\mathfrak{M}$ contains $\mathscr{S}_{e^{\prime}}\left(\mathfrak{A}_{b^{\prime}}\right)$, we have that $\mathfrak{M}=\mathfrak{U}_{b^{\prime}}$, where $e^{\prime}=t(\mathfrak{M})$ and $b^{\prime}=p\left(e^{\prime}\right)$. This is a contradiction. Therefore $j_{e}^{1}(\mathfrak{R})$ must be a proper ideal of $J_{e}^{1}\left(\mathfrak{A}_{b}\right)$ for any $e \in c l(t(S)) \cap \hat{F}_{b, 0}^{b}(b=p(e))$. Hence, for any $e \in \operatorname{cl}(t(S)) \cap \hat{F}_{b, 0}^{b}$, there exists a maximal ideal $\mathfrak{M} \in C L(S)$ such that $t(\mathfrak{M})=e$. This completes the proof of Lemma 5.4 .

Proof of Proposition 5.3. Note that the map $t: \hat{A}^{*} \rightarrow E$ is surjective. We define $\sigma: E \rightarrow E^{\prime}$ by $\sigma(t(\mathfrak{M}))=t^{\prime}(\Phi(\mathfrak{M}))$. First we shall prove that $\sigma$ is well defined. Note that $\sigma=\sigma_{1}$ on $\hat{E}_{1}$ by the definition, and $p^{\prime}\left(\sigma(t(\mathfrak{M}))=\bar{\sigma}(p(t(\mathfrak{M})))\right.$ for any $\mathfrak{M} \in A^{*}$. By the same argument as the proof of [1], Proposition 3.2, we see that $\hat{F}_{b, 0}^{b}$ is discrete in $F_{b}$ for each $b \in B$. Thus, for any $e \in E-\hat{E}_{1}$, there exists a closed neighborhood $D$ of $e$ in $F_{b}$ such that $D \cap \hat{F}_{b, 0}^{b}=\{e\}$, where $b=p(e)$. Let

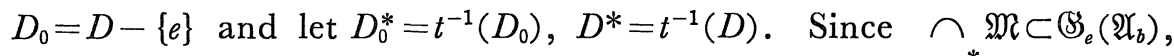
$C L\left(D_{0}^{*}\right)=D^{*}$.

Together with Lemma 5.4, we have that $c l\left(\sigma_{1}\left(D_{0}\right)\right)=c l\left(\sigma_{1}\left(t\left(D_{0}^{*}\right)\right)\right)$ $=\operatorname{cl}\left(t^{\prime}\left(\Phi\left(D_{0}^{*}\right)\right)\right)=t^{\prime}\left(C L\left(\Phi\left(D_{0}^{*}\right)\right)\right)=t^{\prime}\left(\Phi\left(C L\left(D_{0}^{*}\right)\right)\right)=t^{\prime}\left(\Phi\left(D^{*}\right)\right)$. Since $\sigma_{1}$ is homeomorphic, $c l\left(\sigma\left(D_{0}\right)\right) \cap \hat{F}_{\bar{\sigma}(b), 0}^{\bar{\sigma}(b)}$ consist of a point $e^{\prime}$. Thus $t^{\prime}\left(\Phi\left(D^{*}\right)\right) \cap \hat{F}_{\sigma(b), 0}^{\sigma(b)}=\left\{e^{\prime}\right\}$. Therefore $\sigma$ is a well defined bijective map. It is clear by the definition that $p^{\prime} \circ \sigma=\bar{\sigma} \circ p$. By Lemma 5.4, $\sigma$ is homeomorphic, and this completes the proof of Proposition 5.3.

Lemma 5. 5. (Cf. Koriyama, Maeda and Omori [4], Lemma 5.1). 
Let $X \in \mathfrak{X}(E ; p)$ and $e \in E_{1}, b=p(e)$. Then $X_{e} \neq 0$ if and only if $\left[p_{*}(X)\right.$, $\mathfrak{X}(B)]+\overline{\mathfrak{S}}(b)=\mathfrak{X}(B)$ or $\left[X, \mathfrak{A}_{b}\right]+\mathfrak{S}(e)_{b}=\mathfrak{N}_{b}$.

Proof. From [1], Lemma 5.2, $p_{*}(X)_{b} \neq 0$ if and only if $\left[p_{*}(X)\right.$, $\mathfrak{X}(B)]+\overline{\mathfrak{S}}(b)=\mathfrak{X}(B)$. We assume that $p_{*}(X)_{b}=0$. By the same argument as the proof of Lemma 3.5, we see that if $X_{e} \neq 0$, then $\left[X, \mathfrak{X}_{b}\right]$ $+\mathfrak{\Im}(e)_{b}=\mathfrak{N}_{b}$. Now assume that $\left[X, \mathfrak{N}_{b}\right]+\mathfrak{S}(e)_{b}=\mathfrak{N}_{b}$ but $X_{e}=0$. Let $\widetilde{\Im}(e)_{b}^{1}=\left\{Z \in \mathfrak{A}_{b} ;((\operatorname{Ad} Y) Z)_{e}=0\right.$ for $\left.Y \in \mathfrak{X}_{F}(E ; p)\right\}$ be a subalgebra of $\mathfrak{Q}_{b}$. It is easy to see that $[X, Z] \in \mathfrak{S}(e) \frac{1}{b}$ for any $Z \in \mathfrak{J}(e) \frac{1}{b}$. Note that $\mathfrak{J}(e)_{b}^{\frac{1}{b}}$ is finite codimensional in $\mathfrak{A}_{b}$.

Let ad $X: \mathfrak{A}_{b} \rightarrow \mathfrak{Y}_{b}$ be a map defined by $(a d X)(Z)=[X, Z]$ for $Z \in \mathfrak{A}_{b}$. Then the map ad $X$ induces a map $A(X)_{*}: \mathfrak{U}_{b} / \mathfrak{\Im}(e) \frac{1}{b} \rightarrow \mathfrak{U}_{b} /$ $\mathfrak{F}(e)_{b}^{1}$. Since $\left[X, \mathfrak{A}_{b}\right]+\mathfrak{S}(e)_{b}=\mathfrak{A}_{b}, A(X)_{*}$ is epimorphic. Since $A(X)_{*}$ is an endmorphism of the finite dimensional vector space $\mathfrak{A}_{b} / \mathfrak{\Im}(e) \frac{1}{b}$, $A(X)_{*}$ is isomorphic. Let $\{X\}$ be the equivalence class of $X$ in $\mathfrak{A}_{b} /$ $\mathfrak{J}(e)_{b}^{\frac{1}{b}}$. Then $A(X)_{*}\{X\}=0$, hence $X \in \mathfrak{\mathcal { S }}(e)_{b}^{\frac{1}{b}}$. Therefore $\left[X,\left(\mathfrak{U}_{b}\right)_{e}\right] \subset$ $\mathfrak{\Im}(e)_{b}^{1}$, where $\left(\mathfrak{U}_{b}\right)_{e}=\left\{Y \in \mathfrak{A}_{b} ; Y_{e}=0\right\}$. This means that $A(X)_{*}\left(\left(\mathfrak{H}_{b}\right)_{e} /\right.$ $\left.\mathfrak{S}(e)_{b}^{\frac{1}{b}}\right)=0$, and $\left(\mathfrak{U}_{b}\right)_{e}=\mathfrak{J}(e)_{b}^{\frac{1}{b}}$. Since $\left(\mathfrak{U}_{b}\right)_{e} \supseteq \mathfrak{S}_{(}(e)_{b}^{\frac{1}{b}}$, this is a contradiction. This completes the proof of Lemma 5.5.

Let $\mathfrak{X}(E ; p)_{e}=\left\{X \in \mathfrak{X}(E ; p) ; X_{e}=0\right\}$ for each $\mathrm{e} \in E$. From Propositions 5.2, 5.3 and Lemma 5.5, we have the following.

Proposition 5.6. The Lie algebra isomorphism $\Phi: \mathfrak{X}(E ; p) \rightarrow \mathfrak{X}\left(E^{\prime} ; p^{\prime}\right)$ induces a Lie algebra isomorphism $\Phi: \mathfrak{X}(E ; p)_{e} \rightarrow \mathfrak{X}\left(E^{\prime} ; p^{\prime}\right)_{\sigma(e)}$ for each $e \in E$.

Let $V$ be a product $\Gamma$-module $V_{1} \times V_{2}$ of a finite group $\Gamma$ such that $V^{\Gamma}=\{0\}$, and $p_{1}: V \rightarrow V_{1}$ the natural projection. Let $\left\{\eta_{1}, \ldots, \eta_{s}\right\}$ be a minimal set of homogeneous generators for $R\left[V_{2}\right]_{0}^{\Gamma}$. Here $R\left[V_{2}\right]_{0}^{\Gamma}$ is the algebra of $\Gamma$-invariant polynomials which vanish at 0 . Let $\left\{y_{1}, \ldots, y_{m}\right\}$ be a canonical coordinate of $V_{2}$ such that $\Gamma$ acts orthogonally on this coordinate. We can assume $\eta_{1}=y_{1}^{2}+\cdots+y_{m}^{2}$. Let $C_{\Gamma}^{\infty}(V)$ denote the set of all $\Gamma$-invariant smooth functions on $M$.

Lemma 5.7. Let $g$ be a $\Gamma$-invariant continuous function on $V$ such that $\eta_{i} g \in C_{\Gamma}^{\infty}(V)$ for $i=1, \ldots, s$. Then $g$ is a $\Gamma$-invariant smooth function. 
Proof. If $s=1$, by using Taylor expansion, it is easy to prove that $g$ is a smooth function. We consider the case that $s \geqq 2$. Put $h_{i}=\eta_{i} g$. Then we see that $h_{1} \eta_{2}=h_{2} \eta_{1}$. Put $G=h_{1} \eta_{2}$. Let $T_{a} G$ be a formal Taylor expansion at $a \in V$. Then $T_{a} G=\eta_{2} T_{a}\left(h_{1}\right)=\eta_{1} T_{a}\left(h_{2}\right)$. Since $\left\{\eta_{1}, \ldots, \eta_{s}\right\}$ is a minimal set of homogeneous generators for $R\left[V_{2}\right]_{0}^{\Gamma}$, there exists a smooth function $h_{1}^{\prime}$ on $V$ such that $h_{1}=\eta_{1} h_{1}^{\prime}$. Then $g$ is a smooth function.

Theorem 5.8. There exists a Lie algebra isomorphism $\Phi: \mathfrak{X}(E ; p) \rightarrow$ $\mathfrak{X}\left(E^{\prime} ; p^{\prime}\right)$ if and only if there exists a fibration preserving diffeomorphism $\sigma: E \rightarrow E^{\prime}$ such that $\Phi=\sigma_{*}$.

Proof. Using Proposition 5.6 and Lemma 5.7, we can prove Theorem 5.8 by the same argument as in [1], §5.

\section{§6. Reflection Groups}

Let $V$ be an $n$-dimensional product $\Gamma$-module $V_{1} \times V_{2}$ of a finite group $\Gamma$. Let $\Gamma_{v}$ denote the isotropy subgroup of $\Gamma$ at $v \in V$. Let $V^{(1)}=\left\{v \in V ; \Gamma_{v}\right.$ is a cyclic group of order two generated by a reflection of $V\}$. Let $\Gamma_{1}$ be the subgroup of $\Gamma$ generated by reflections $\left\{\gamma \in \Gamma_{v}\right.$; $\left.v \in V^{(1)}\right\} . \quad \Gamma_{1}$ is a normal subgroup of $\Gamma$ which is a reflection group. Let $\Gamma_{1}^{i}(i=1,2)$ be the subgroup of $\Gamma_{1}$ generated by reflections $\{\gamma \in$ $\left.\Gamma_{v} ; v \in V^{(1)} \cap V_{i}\right\}$. Then $\Gamma_{1}=\Gamma_{1}^{1} \times \Gamma_{1}^{2}$. Since $\Gamma_{1}$ is a reflection subgroup, there is a homogeneous minimal set of generators $\left\{\theta_{1}, \ldots, \theta_{n}\right\}$ for $R[V]_{0}^{\Gamma_{1}}$ (see Bourbaki [3], Chapitre $V, \S 5$, Théorème 3]). Let $n_{i}=$ $\operatorname{dim} V_{i}$ for $i=1,2$. We can assume that $\left\{\theta_{1}, \ldots, \theta_{n_{1}}\right\}$ and $\left\{\theta_{n_{1}+1}, \ldots\right.$, $\left.\theta_{n}\right\}$ are homogeneous minimal sets of generators for $R\left[V_{1}\right]_{0}^{\Gamma_{1}^{1}}$ and $R\left[V_{2}\right]_{0}^{\Gamma_{1}^{2}}$ respectively. Let $\theta^{1}=\left(\theta_{1}, \ldots, \theta_{n_{1}}\right): V_{1} \rightarrow R^{n_{1}}, \theta^{2}=\left(\theta_{n_{1}+1}, \ldots, \theta_{n}\right)$ : $V_{2} \rightarrow R^{n_{2}}$ and $\theta=\left(\theta^{1}, \theta^{2}\right): V \rightarrow R^{n}$ be polynomial maps. Let $\bar{\theta}^{1} ; V_{1} / \Gamma_{1}^{1} \rightarrow R^{n_{1}}$, $\bar{\theta}^{2}: V_{2} / \Gamma_{1}^{2} \rightarrow R^{n_{2}}$ and $\bar{\theta}: V / \Gamma_{1} \rightarrow R^{n}$ be the induced orbit maps which are embeddings. Since $\Gamma_{1}$ is a normal subgroup of $\Gamma$, the $\Gamma$-action on $V$ induces an action $\Psi_{0}: \bar{\Gamma} \times V / \Gamma_{1} \rightarrow V / \Gamma_{1}$, where $\bar{\Gamma}$ is a factor group $\Gamma / \Gamma_{1}$ 。

Lemma 6.1. There exists a linear action $\Psi: \bar{\Gamma} \times R^{n} \rightarrow R^{n}$ such that 
(1) $\Psi(\gamma, \bar{\theta}(X))=\vec{\theta}\left(\Psi_{0}(\gamma, x)\right)$ for $\gamma \in \bar{\Gamma}, x \in V / \Gamma_{1}$, and $\bar{\theta}: V / \Gamma_{1} \rightarrow R^{n}$ is a $\bar{\Gamma}$-equivariant embedding.

(2) $\Psi\left(\gamma, \bar{\theta}^{i}(x)\right)=\bar{\theta}^{i}\left(\Psi_{0}(\gamma, x)\right)$ for $\gamma \in \bar{\Gamma}, x \in V_{i} / \Gamma_{1}^{i}(i=1,2)$, and $\bar{\theta}^{i}$ : $V_{i} / \Gamma_{1}^{i} \rightarrow R^{n_{i}}$ is a $\bar{\Gamma}$-equivariant embedding.

Proof. Since $\Gamma_{1}$ is a normal subgroup of $\Gamma, \bar{\Gamma}$ acts on $R[V]_{0}^{\Gamma_{1}}$ by $(\gamma \circ f)(v)=f\left(\gamma^{-1} \circ v\right)$ for $f \in R\{V]_{0}^{\Gamma_{1}}, \gamma \in \bar{\Gamma}, v \in V$. We can choose a homogeneous minimal set of generators for $R[V]_{0}^{\Gamma_{1}}$ such that $\gamma \circ \theta_{i}=$ $\Sigma_{\operatorname{deg} \theta_{i}=\operatorname{deg} \theta_{j}} a_{i j}(\gamma) \theta_{j}$ with $\left(a_{i j}(\gamma)\right) \in G L(n, R)$ for $\gamma \in \bar{\Gamma}(\operatorname{cf}$. Schwarz [8], Lemma 8.1). We can assume that $a_{i j}=0$ if $1 \leqq \mathrm{i} \leqq n_{1}, n_{1}+1 \leqq j \leqq n$ or $1 \leqq j \leqq n_{1}, n_{1}+1 \leqq i \leqq n$. Let $\left\{e_{1}, \ldots, e_{n}\right\}$ be a canonical basis of $R^{n}$. We define a linear action $\Psi: \bar{\Gamma} \times R^{n} \rightarrow R^{n}$ by $\Psi\left(\gamma, \sum_{j=1}^{n} x_{j} e_{j}\right)=\sum_{i, j=1}^{n} a_{i j}\left(\gamma^{-1}\right) x_{j} e_{i}$. Then we can check that the action satisfies (1) and (2). This completes the proof of Lemma 6.1.

Let $p: V / \Gamma \rightarrow V_{1} / \Gamma$ and $\rho: R^{n} / \bar{\Gamma} \rightarrow R^{n_{1}} / \bar{\Gamma}$ be maps induced from the natural projections $V \rightarrow V_{1}$ and $R^{n} \rightarrow R^{n_{1}}$ respectively. We can regard $p$ and $\rho$ as fibrations over smooth orbifolds. Since $\bar{\theta}: V / \Gamma_{1} \rightarrow R^{n}$ is a $\Gamma$-equivariant embedding by Lemma 6.1 , we have an induced embedding $\overline{\bar{\theta}}: V / \Gamma \rightarrow R^{n} / \bar{\Gamma}$. It is easy to see that the induced map $\bar{\theta}^{*}: C^{\infty}$ $\left(R^{n} / \bar{\Gamma}\right) \rightarrow C^{\infty}(V / \Gamma)$ is epimorphic. We define $\bar{\theta}^{*}: \mathfrak{D}\left(R^{n} / \bar{\Gamma}\right) \rightarrow \mathfrak{D}(V / \Gamma)$ by $\overline{\bar{\theta}}^{*}(X)\left(\overline{\bar{\theta}}^{*}(f)\right)=X(f) \circ \overline{\bar{\theta}}$ for $X \in \mathfrak{D}\left(R^{n} / \bar{\Gamma}\right), f \in C^{\infty}\left(R^{n} / \bar{\Gamma}\right)$. Since $\bar{\theta}(V / \Gamma)$ is the closure of an open set in $R^{n} / \bar{\Gamma}$, we see that $\bar{\theta}^{*}$ is a well defined Lie algebra homomorphism. Clearly $\bar{\theta}^{*}$ induces a Lie algebra homomorphism $\overline{\bar{\theta}}^{*}: \mathfrak{D}\left(R^{n} / \bar{\Gamma} ; \rho\right) \rightarrow \mathfrak{D}(V / \Gamma ; p)$.

Let $\left\{\eta_{1}, \ldots, \eta_{k}\right\}$ be a minimal set of generators for $R\left[R^{n}\right]_{0}^{\bar{\Gamma}}$ such that $\left\{\eta_{1}, \ldots, \eta_{k_{1}}\right\}\left(k_{1} \leqq k\right)$ is a minimal set of generators for $R\left[R^{n_{1}}\right]_{0}^{\bar{\Gamma}}$. Let $\bar{\eta}: R^{n} / \bar{\Gamma} \rightarrow R^{k}$ be the orbit map of a polynomial map $\eta=\left(\eta_{1}, \ldots, \eta_{k}\right)$ : $R^{n} \rightarrow R^{k}$. Let $\nu=\left(\nu_{1}, \ldots, \nu_{k}\right): V \rightarrow R^{k}$, where $\nu_{i}=\eta_{i} \circ \theta$. Since $\vec{\theta}$ and $\dot{\eta}$ are smooth embeddings, $\nu(V)=\bar{\eta}(\bar{\theta}(V / \Gamma))$ is diffeomorphic to $V / \Gamma$. Let $r_{i} \in R\left[y_{1}, \ldots, y_{k}\right], i=1, \ldots, l$, be the generators of the ideal $I$ of algebraic relations among $\eta_{1}, \ldots, \eta_{k}$. Let $S$ be a subset of $R^{n}$ and $I^{\infty}(S)$ denote the ideal in $C^{\infty}\left(R^{n}\right)$ which vanish on $S$. For a smooth vector field $X$ on $R^{n}$, we say that $X$ is tangent to $S$ if $X$ preserves the ideal $I^{\infty}(S)$. 
Lemma 6.2. Let $X$ be a polynomial vector field on $R^{k}$. Then

(1) $X$ is tangent to $\eta\left(R^{n}\right)$ if and only if $X\left(r_{i}\right) \in I, i=1, \ldots, l$.

(2) $X$ is tangent to $\eta\left(R^{n}\right)$ if and only if $X$ is tangent to $\nu(V)$.

Proof. Since the ring of polynomial functions on $\eta\left(R^{n}\right)$ can be identified with $R\left[y_{1}, \ldots, y_{k}\right] / I$, we see (1). Since $\nu(V)$ contains an open set in $R^{n}$, we can prove that $I$ is also the ideal of algebraic relations among $\nu_{1}, \ldots, \nu_{k}$, and (2) follows.

Let $D\left(\eta\left(R^{n}\right)\right)$ be the Lie algebra of polynomial vector fields on $R^{k}$ which tangent to $\eta\left(R^{n}\right)$. By the same argument as in Schwarz [8, Proposition 6.14], we see the following.

Proposition 6. 3. (1) $\cong\left(\eta\left(R^{n}\right)\right)=C^{\infty}\left(\eta\left(R^{n}\right)\right) \cdot D\left(\eta\left(R^{n}\right)\right)$. (2) $\mathscr{D}(\nu(V))=C^{\infty}(\nu(V)) \cdot D(\nu(V))$.

Proposition 6.4. The Lie algebra homomorphism $\bar{\theta}^{*}: \mathfrak{D}\left(R^{n} / \bar{\Gamma}\right) \rightarrow \mathfrak{D}$ $(V / \Gamma)$ is epimorphic.

Proof. By Lemma 6.2 $D\left(\eta\left(R^{n}\right)\right)=D(\nu(V))$. Let $j: \nu(V) \subseteq \eta\left(R^{n}\right)$ be the inclusion. Then the induced map $j^{*}: C^{\infty}\left(\eta\left(R^{n}\right)\right) \rightarrow C^{\infty}(\nu(V))$ is epimorphic. Since $\bar{\theta}^{*}$ is identified with a homomorphism $\mathfrak{S}\left(R^{n} / \bar{\Gamma}\right)$ $=\mathfrak{D}\left(\eta\left(R^{n}\right)\right) \stackrel{j *}{\longrightarrow} \mathfrak{D}(\nu(V))=\mathfrak{D}(V / \Gamma), \bar{\theta}^{*}$ is epimorphic from Proposition 6. 3. This completes the proof of Proposition 6. 4 .

Note that the maps $p: V / \Gamma \rightarrow V_{1} / \Gamma$ and $\rho: R^{n} / \bar{\Gamma} \rightarrow R^{n_{1}} / \bar{\Gamma}$ induce Lie algebra homomorphisms $p_{*}: \mathfrak{D}(V / \Gamma ; p) \rightarrow \mathfrak{D}\left(V_{1} / \Gamma\right)$ and $\rho_{*}: \mathfrak{D}\left(R^{n} / \bar{\Gamma} ; \rho\right)$ $\rightarrow \mathfrak{D}\left(R^{n_{1}} / \bar{\Gamma}\right)$. We set $\mathfrak{D}_{F}(V / \Gamma ; p)=\operatorname{ker} p_{*}$ and $\mathfrak{D}_{F}\left(R^{n} / \bar{\Gamma} ; \rho\right)=\operatorname{ker} \rho_{*}$. Then $\mathfrak{D}_{F}(V / \Gamma ; p)$ is a $C^{\infty}(V / \Gamma)$-module and $\mathfrak{D}_{F}\left(R^{n} / \bar{\Gamma} ; \rho\right)$ is a $C^{\infty}\left(R^{n} / \bar{\Gamma}\right)$-module respectively. Let $q: R^{k} \rightarrow R^{k_{1}}$ be the natural projection $q$ induces a Lie algebra homomorphism $q_{*}: \mathfrak{D}\left(R^{k} ; q\right) \rightarrow \mathscr{D}\left(R^{k}\right)$. Let $D_{F}\left(\eta\left(R^{n}\right) ; q\right)=\operatorname{ker} q_{*} \cap D\left(\eta\left(R^{n}\right)\right) \quad$ and $\quad D_{F}(\nu(V) ; q)=\operatorname{ker} q_{*} \cap D(\nu(V))$. By the same argument as in Proposition 6.3 and Proposition 6.4, we have the following.

Proposition 6.5. (1) $\mathfrak{D}_{F}(\eta(V) ; q)=C^{\infty}(\eta(V)) \cdot D_{F}(\eta(V) ; q)$.

(2) $\mathfrak{S}_{F}(\nu(V) ; q)=C^{\infty}(\nu(V)) \cdot D_{F}(\nu(V) ; q)$. 
(3) The Lie algebra homomorphism $\bar{\theta}^{*}: \mathfrak{D}_{F}\left(R^{n} / \bar{\Gamma} ; \rho\right) \rightarrow \mathfrak{D}_{F}(V / \Gamma ; p)$ is epimorphic.

Combining Propositions 6.4 and 6.5, we have.

Corollary 6.6. The Lie algebra homomorphism $\overline{\bar{\theta}}^{*}: \mathfrak{D}\left(R^{n} / \bar{\Gamma} ; \rho\right) \rightarrow$ $\mathfrak{D}(V / \Gamma ; p)$ is epimorphic.

Lemma 6.7 If $V^{(1)}$ is not empty, then there exists a smooth vector field $X \in \mathfrak{D}(V / \Gamma ; p)$ such that $X_{\pi(0)} \neq 0$, where $\pi: V \rightarrow V / \Gamma$ is the natural projection.

Proof. $\quad V$ is an orthogomal representation with respect to a suitable basis of $V$. Let $W$ be the orthogonal complement of $V^{\Gamma}$. Then $W$ is a $\Gamma$-module. Since $V^{(1)}$ is not empty, $\operatorname{dim} W>0$. Let $\left\{x_{1}, \ldots, x_{p}\right\}$ be a canonical coordinate of $W$. If $\operatorname{dim} W^{\Gamma}>0$, we see that there exists a $\Gamma$-invariant smooth vector field $Y$ on $V$ such that $X=\pi_{*}(Y) \in$ $\mathfrak{D}(V / \Gamma ; p)$ and $X_{\pi(0)} \neq 0$. If $\operatorname{dim} W^{\Gamma}=0$, then we can choose a homogeneous minimal set of generators $\left\{\theta_{1}, \ldots, \theta_{n}\right\}$ for $R[V]_{0}^{\Gamma_{1}}$ such that $\theta_{i}=x_{1}^{2}+\cdots+x_{p}^{2}$ for some $i$. Since $\gamma \circ \theta_{i}=\theta_{i}$ for any $\gamma \in \Gamma$, we see that $\operatorname{dim}\left(R^{n}\right) \bar{\Gamma}>0$. Then we have a $\bar{\Gamma}$-invariant smooth vector field $Z$ on $R^{n}$ such that $X=\pi_{*}^{\prime}(Z) \in \mathfrak{S}\left(R^{n} / \bar{\Gamma} ; p\right)$ and $X_{\pi^{\prime}(0)} \neq 0$, where $\pi^{\prime}: R^{n} \rightarrow R^{n} / \bar{\Gamma}$ is the natural projection. Since $(d \bar{\theta})_{\pi(0)}: \tau_{\pi(0)}(V / \Gamma) \rightarrow \tau_{\pi^{\prime}(0)}\left(R^{n} / \bar{\Gamma}\right)$ is isomorphic, $\theta_{*}(X)_{\pi(0)} \neq 0$. This completes the proof of Lemma 6.7.

Let $\mathfrak{D}(V / \Gamma ; p)(x)=\left\{Y_{x} ; Y \in \mathfrak{D}(V / \Gamma ; p)\right\}$ for $x \in V / \Gamma$.

Corollary 6.8. $\cong(V / \Gamma ; p)(\pi(0))=\{0\}$ if and only if dim $V^{\Gamma=0}$ and $V^{(1)}=\phi$.

Proof. If $V^{(1)}=\phi$, then $\mathfrak{D}(V / \Gamma ; p)=\mathfrak{X}(V / \Gamma ; p)$ by Schwarz [8, Chapter I, Proposition 3.5]. By Lemma $2.2 \mathfrak{X}(V / \Gamma ; p)=\mathfrak{X}_{\Gamma}(V ; \tilde{p})$, where $\tilde{p}: V \rightarrow V_{1}$ is the natural projection. Thus if $V^{(1)}=\phi$ and $\operatorname{dim}$ $V^{\Gamma}=0, \mathfrak{D}(V / \Gamma ; p)(\pi(0))=\{0\}$ from Lemmas 2.3 and 2.5. If $V^{(1)} \neq \phi$, then $D(V / \Gamma ; p)(\pi(0)) \neq\{0\}$ by Lemma 6.7 , and Corollary 6.8 follows. 


\section{§7. Proof of Theorem 0.1 and Theorem 0.2}

Let $p: E \rightarrow B$ be a fibration over a connected smooth orbifold $B$ with generic fibre $F$, a connected smooth manifold. We set $B_{0}=$ $\left\{b \in B ; X_{b}=0\right.$ for any $\left.X \in \mathfrak{D}(B)\right\}, B_{1}=B-B_{0}$ and $\mathfrak{D}(B)_{b}=\{X \in \mathfrak{D}(B)$; $\left.X_{b}=0\right\}$.

Proposition 7.1. For $b \in B_{1}$, there exists a unique infinite codimensional

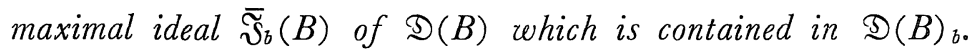

Proof. For $b \in B$, there exist a finite group $\Gamma$ and a linear action $\Gamma \times V \rightarrow V$ on an n-dimensional vector space $V$ such that $V / \Gamma$ is diffeomorphic to an open neighborhood $U$ of $b$. By Lemma 6. 1 and Proposition 6.4, we have a linear action $\bar{\Gamma} \times R^{n} \rightarrow R^{n}$ such that there exists an embedding $\overline{\bar{\theta}}: V / \Gamma \rightarrow R^{n} / \bar{\Gamma}$ and $\bar{\theta}^{*}: \mathfrak{D}\left(R^{n} / \bar{\Gamma}\right) \rightarrow \mathfrak{D}(V / \Gamma)$ is a Lie algebra epimorphism. Using the property of the reflection group $\Gamma_{1}$, the natural group homomorphism $\Gamma \rightarrow \bar{\Gamma}$ has a right inverse. Then it is easy to see that $\bar{\Gamma}$ has no reflection subgroups. Then $\mathscr{D}\left(R^{n} / \bar{\Gamma}\right)$ $=\mathfrak{X}\left(R^{n} / \bar{\Gamma}\right)$. By Corollary 6.6 and Lemma 6.7, there exists a smooth vector field $X \in \mathfrak{S}\left(R^{n} / \bar{\Gamma}\right)$ such that $X_{\pi(0)} \neq 0$.

From [1], Lemma 3.9, there exists a unique infinite codimensional maximal ideal $\mathfrak{N}_{1}$ of $\mathfrak{X}\left(R^{n} / \bar{\Gamma}\right)$ which is contained in $\mathfrak{X}\left(R^{n} / \bar{\Gamma}\right)_{b}$ 。 Then $\mathfrak{R}_{2}=\overline{\bar{\theta}}^{*}\left(\mathfrak{R}_{1}\right)$ is an infinite codimensional maximal ideal of $\mathfrak{D}(V / \Gamma)$ which is contained in $\mathfrak{S}(V / \Gamma)_{b}$ o $\quad \bar{\Im}_{b}(B)=\{X \in \mathfrak{S}(B) ; X=Y$ on a neighborhood of $b$ in $B$ for some $\left.Y \in \mathfrak{N}_{2}\right\}$. As in the proof of [1], Proposition 3.8, we can prove that $\overline{\mathfrak{\Im}}_{b}(B)$ is an infinite codimensional maximal ideal of $\mathfrak{D}(B)$. This completes the proof of Proposition 7.1.

Lemma 7.2. Let $b \in B_{1}$. Then, for $X \in \mathfrak{D}(B), X(b) \neq 0$ if and only if $[X, \mathfrak{D}(B)]+\bar{\Im}_{b}(B)=\mathfrak{D}(B)$.

Proof. We use the same notation as in the proof of Proposition 7. 1. To prove Lemma 7.2, it is sufficient to prove that, for $X \in \mathfrak{D}$ $(V / \Gamma), \quad X_{\pi(0)} \neq 0$ if and only if $[X, \mathfrak{D}(V / \Gamma)]+\mathfrak{R}_{2}=\mathfrak{D}(V / \Gamma)$ where $\pi: V \rightarrow V / \Gamma$ is the natural projection. Note that $\mathfrak{D}\left(R^{n} / \bar{\Gamma}\right)=\mathfrak{X}\left(R^{n} / \bar{\Gamma}\right)$. It follows from [1], Lemma 5.2 that, for $Y \in \mathfrak{D}\left(R^{n} / \bar{\Gamma}\right), Y_{\rho(0)} \neq 0$ if and only if $\left[Y, \mathfrak{S}\left(R^{n} / \bar{\Gamma}\right)\right]+\mathfrak{N}_{1}=\mathfrak{D}\left(R^{n} / \bar{\Gamma}\right)$, where $\rho: R^{n} \rightarrow R^{n} / \bar{\Gamma}$ is the 
natural projection. Let $X \in \mathfrak{D}(V / \Gamma)$ with $X_{\pi(0)} \neq 0$. There exists $Y \in \mathfrak{D}$ $\left(R^{n} / \bar{\Gamma}\right)$ such that $\bar{\theta}^{*}(Y)=X$. Then $Y_{\rho(0)} \neq 0$ and hence $\left[Y, \mathfrak{D}\left(R^{n} / \bar{\Gamma}\right)\right]$ $+\mathfrak{N}_{1}=\mathfrak{D}\left(R^{n} / \bar{\Gamma}\right)$. Since $\bar{\theta}^{*}$ is epimorphic, $[X, \mathfrak{D}(V / \Gamma)]+\mathfrak{N}_{2}=\mathfrak{D}(V / \Gamma)$. Conversely, suppose that $[X, \mathfrak{D}(V / \Gamma)]+\mathfrak{N}_{2}=\mathfrak{D}(V / \Gamma)$. Let $Y \in \mathfrak{D}$ $\left(R^{n} / \bar{\Gamma}\right)$ such that $\bar{\theta}^{*}(Y)=X$. It is easy to see that $\mathscr{D}\left(R^{n} / \bar{\Gamma}\right)=[Y, \mathscr{D}$ $\left.\left(R^{n} / \bar{\Gamma}\right)\right]+\mathfrak{N}_{1}$. Then $Y_{\rho(0)} \neq 0$. Hence $X_{\pi(0)} \neq 0$, and this completes the proof of Lemma 7.2.

Proof of Theorem 0.1. Using the result of [1], we see that (2) implies (3). By Schwarz [8], Corollary 1.7, if $\sigma: B \rightarrow B^{\prime}$ is diffeomorphic, then $\sigma$ is strata preserving. Then we see that (3) implies (2). Assume that there exists a Lie algebra isomorphism $\Phi: \mathfrak{D}(B) \rightarrow \mathfrak{D}\left(B^{\prime}\right)$. As in [1], §5, using Proposition 7.1, and Lemma 7.2, we can prove that there exists a diffeomorphism $\sigma: B \rightarrow B^{\prime}$ such that $\Phi=\sigma_{*}$. This completes the proof of Theorem 0.1 .

Proof of Theorem 0.2. By Theorem 5.8, (2) implies (3). By Schwarz [8], Corollary 1.7, (3) implies (2). Assume that there exists a Lie algebra isomorphism $\Phi: \mathfrak{D}(E ; p) \rightarrow \mathfrak{D}\left(E^{\prime} ; p^{\prime}\right)$. As the proof of Theorem 5.7, using Corollary 6.6 and Theorem 0.1 , we can prove that there exists a fibration preserving diffeomorphism $\sigma: E \rightarrow E^{\prime}$ such that $\Phi=\sigma_{*}$. This complete the proof of Theorem 0.2.

\section{References}

[1] Abe, K., Pursell-Shanks type theorem for orbit spaces of G-maniflods, Publ. Res. Inst. Math. Sci. Kyoto Univ. 18 (1982), 685-702.

[2] Bierstone, E., Lifting isotopies from orbit spaces, Topology 14 (1975), 245-252.

[3] Bourbaki, N., Groupes et Algèbres de Lie, Paris Herman, 1958.

[4] Koriyama, A., Maeda, Y. and Omori, H., On Lie algebras of vector fields, Trans. Amer. Math. Soc. 226 (1977), 89-117.

[5] — On Lie algebras of vector fields on expansive sets, Japan J. Math. 3 (1977) $57-88$.

[6] Omori, H., Infinite Dimensional Lie Transformation Groups, Springer Lecture Notes in Math. 427, Springer-Verlag, (1974).

[ 7 ] Satake, I., The Gauss-Bonnet theorem for V-manifolds, J. Math. Soc. Japan 9 (1957), 464-492.

[8] Schwarz, G. W., Lifting smooth homotopies of orbit spaces, Inst. Hautes Etudes Sci. Publ. Math. 51 (1980), 37-135.

[9] Sternberg, S., Local contractions and a theorem of Poincaré, Amer. J. Math. 79(1957), 809-824.

[10] Thurston, W., The geometry and topology of 3-manifolds, to be published by Princeton Univ. Press. 
